\title{
Effects of a single-dose administration of Bowman-Birk inhibitor concentrate on anti-proliferation and inhabitation of metastasis in M5076 ovarian sarcoma-bearing mice
}

\author{
NORITAKA SAKURAI ${ }^{1}$, KAZUYUKI SUZUKI ${ }^{1}$, YUKI SANO ${ }^{1}$, TERUYOSHI SAITO ${ }^{1}$, HISASHI YOSHIMURA ${ }^{1}$, \\ YUKIE NISHIMURA $^{1}$, TOMOHIRO YANO ${ }^{2}$, YASUYUKI SADZUKA ${ }^{3}$ and RYUJI ASANO ${ }^{1}$ \\ ${ }^{1}$ Department of Veterinary Medicine, College of Bioresource Sciences, Nihon University, Fujisawa, Kanagawa 252-8510; \\ ${ }^{2}$ Department of Food Science Research for Health, National Institute of Health and Nutrition, Shinjuku, Tokyo 162-8636; \\ ${ }^{3}$ School of Pharmacy, Iwate Medical University, Shiwa-gunn, Iwate 028-3694, Japan
}

Received June 17, 2008; Accepted September 10, 2008

DOI: 10.3892/mmr_00000048

\begin{abstract}
The present study was designed to investigate the effects of Bowman-Birk inhibitor concentrate (BBIC) on antiproliferation, up-regulation of Connexin 43 (Cx43) expression and inhabitation of hepatic metastasis in mice with M5076 ovarian sarcoma. M5076 ovarian sarcomas ( $1 \times 10^{6}$ cells/animal) were subcutaneously transplanted into the back of $\mathrm{BDF}_{1}$ mice. The 'pre-treated' $(n=10)$ and 'post-treated' $(n=10)$ groups were fed a standard diet (CE-2) compounded with $0.5 \%$ BBI from 3 weeks before and from the day of tumor inoculation, respectively, until 4 weeks after tumor inoculation. The 'control group' $(\mathrm{n}=10)$ was fed CE-2 alone. Relative tumor weights in the pre- $(0.013 \pm 0.010)$ and post- $(0.012 \pm 0.013)$ treated groups were significantly reduced by 30.0 and $32.5 \%$, respectively, as compared to the control group $(0.040 \pm 0.022$, $\mathrm{p}<0.01)$. The relative densities of $\mathrm{Cx} 43$ mRNA and $\mathrm{Cx} 43$ protein were significantly higher in the BBIC-treated groups than in the control group. The median numbers of macroscopic spontaneous metastases were significantly lower in the pre$(1.0 \pm 2.3)$ and post- $(1.9 \pm 3.6)$ treated groups than in the control group $(71.4 \pm 97.3)$. These results suggest that BBIC reduces proliferation as a result of increased expression of $\mathrm{Cx} 43$ genes in mice with M5076 ovarian sarcoma. In addition, BBIC inhibits hepatic metastasis in M5076-bearing mice.
\end{abstract}

\section{Introduction}

There are several components which have been isolated from soybeans and have been investigated as cancer preventive agents (1). The Bowman-Birk inhibitor (BBI), which was

Correspondence to: Dr Kazuyuki Suzuki, Department of Veterinary Medicine, College of Bioresource Sciences, Nihon University, 1866 Kameino, Fujisawa, Kanagawa 252-8510, Japan

E-mail:kazuyuki@rakuno.ac.jp

Key words: anti-proliferation, Bowman-Birk inhibitor, Connexin 43, hepatic metastasis, M5076 ovarian sarcoma identified by Bowman in 1946 (2) and purified by Birk in 1961 (3), is an 8-kDa polypeptide derived from soybeans that has demonstrated chemopreventive activities in a variety of in vitro and in vivo carcinogenesis assay systems (4). This polypeptide is a serine protease inhibitor that can inhibit trypsin and chymotrypsin activities (5). Functionally, BBI is a double-headed serine protease inhibitor that inhibits both trypsin- and chymotrypsin-like serine proteases. It reduces the activities of trypsin, chymotrypsin, elastase, cathepsin G, chymase, serine protease-dependent matrix metalloproteinases, urokinase protein activator, mitogen-activated protein kinase and PI3 kinase $(6,7)$. Chymotrypsin inhibition is preferentially associated with the anti-carcinogenic effects of BBI $(4,6,8)$.

Chen et al (9) demonstrated that BBI specifically and potently inhibits proteasomal chymotrypsin-like activity in vitro and in vivo in MCF-7 breast cancer cells through the accumulation of ubiquitinated proteins and the proteasome substrates p21 and p27, accompanied by the down-regulation of cyclin D1 and E. These findings suggest that the chemopreventive effect of BBI is also associated with proteasome inhibition, and provide further evidence that soybean products containing active BBI have the potential to advance as chemopreventive agents. Zhang et al (10) reported that Connexin 43 (Cx43) suppressed the G1-S cell cycle transition by increasing the level of p27. A previous study demonstrated that the anticarcinogenic effects of BBI induced negative growth caused by restoring the expression of $\mathrm{Cx} 43$ genes in M5076 ovarian sarcoma-bearing mice (11). Thus, BBI has an effect on $\mathrm{Cx} 43$, and it may be possible to establish potential cancer chemoprevention and therapy based on $\mathrm{Cx}$ functions. From these criteria, BBI may be a potential agent for cancer chemoprevention and therapy, as it stimulates both the biosynthesis of Cx43 and suppresses the degradation of Cx43 protein.

$\mathrm{BBI}$ concentrate (BBIC) is an extract of soybeans enriched in BBI, which has attenuated trypsin-inhibitory activity (8). Importantly, BBIC has attained investigational new drug (IND) status with the FDA. Trials to evaluate it as an anticarcinogenic agent in human populations have been ongoing for over a decade, with encouraging results indicating it has clinical efficacy and minimal toxicity. For example, a Phase I trial of BBIC in 19 male subjects with benign prostatic 
hyperplasia and lower urinary tract symptoms was conducted. In the benign prostatic hyperplasia patients administered BBIC, reduced prostate volume, reductions in serum levels of prostate-specific antigen and triglycerides and increased sexual function were observed (12). In a Phase IIa trial $(13,14)$, changes in protease activity in oral mucosal cells after BBIC treatment correlated with changes in protein levels.

As a result, the present study was designed to investigate the effects of BBIC, specifically $10 \%$ crude type BBI, on tumor anti-proliferation and up-regulation of $\mathrm{Cx} 43$ expression in mice with M5076 ovarian sarcoma. The effect of BBIC on the inhibition of hepatic metastasis in the mice was also investigated.

\section{Materials and methods}

Chemicals and antibodies. BBIC (10\% BBI) was obtained from Dr K. Takamatsu (Fujioil Co., Osaka, Japan). The primary antibodies to mouse $\mathrm{Cx} 43$ (Chemicon International Inc., Temecula, CA, USA) and mouse B-actin (Sigma-Aldrich Co., MO, USA) and all the respective secondary anti-mouse IgG-conjugated anti-bodies (Beckman Coulter Co., Cedex, France) were purchased from the companies indicated. M5076 ovarian sarcoma cells were kindly provided by Dr T. Tashiro (Japanese Foundation for Cancer Research, Tokyo, Japan).

Animals. Male C57BL/6 and $\mathrm{BDF}_{1}\left(\mathrm{~F}_{1}\right.$ from $\mathrm{C} 57 \mathrm{BL} / 6$ female and DBA/2 male) mice, 5 weeks of age and 20-25 g in weight, were obtained from Clea Japan Inc. (Tokyo, Japan) and housed in a room maintained at $22 \pm 1^{\circ} \mathrm{C}$ and $55.5 \%$ relative humidity. The mice were given free access to regular chow pellets (CE-2, Clea Japan Inc.) and fresh water. The animals were acclimatized for 1 week before use and maintained at standard conditions throughout.

In vivo tumor experiment. The C57BL/6 mice were used for animal passage of M5076 cells, and $\mathrm{BDF}_{1}$ mice were used for in vivo experiments. For animal passage, M5076 ovarian sarcomas $\left(1 \times 10^{6}\right.$ cells/animal $)$ were transplanted intraperitoneally into $\mathrm{C} 57 \mathrm{BL} / 6$ mice. The ascitic cells were collected on the 14th day after transplantation $(11,15)$.

M5076 ovarian sarcomas $\left(1 \times 10^{6}\right.$ cells/animal $)$ were subcutaneously transplanted into the back of $\mathrm{BDF}_{1}$ mice. The 'pre-treated' $(n=10)$ and 'post-treated' $(n=10)$ groups were fed a standard diet (CE-2, Crea Japan) compounded with BBIC $(0.5 \% \mathrm{BBI})$ from 3 weeks before and from the day of tumor inoculation, respectively, until the end of the experiment. The 'control group' $(n=10)$ was fed a standard diet alone during the entire experimental period. All mice were sacrificed 4 weeks after tumor inoculation. The relative tumor (tumor/body weight) and liver (liver/body weight) weights were demonstrated to be indicators of proliferation and hepatic metastasis, respectively $(11,15)$. Solid tumor and liver were therefore extracted and weighed immediately after sacrifice.

Cx43 mRNA and Cx43 protein analysis. The expression levels of Cx43 mRNA and Cx43 proteins were measured by RT-PCR and Western blotting, respectively. Total RNA was extracted with a lysis reagent (Qiazol, Qiagen Inc., Valencia, CA, USA) according to the manufacturer's instructions. RNA extract was stored at $-80^{\circ} \mathrm{C}$ until use. The expression level of glycer- aldehyde-3-phosphate dehydrogenase (GAPDH) was used as an internal control. Total RNA ( $3 \mu \mathrm{g})$ was used for first-strand cDNA synthesis with SuperScript Reverse Transcriptase (Invitrogen Co., Carlsbad, CA, USA). For amplification of the target genes, the following primer pairs were used: GAPDH forward primer 5'-AAGGCTGAGAACGGGAAACT-3' and reverse 5'-GGAGGCATTGCTGACAATCT-3' for the amplification of a 249-bp fragment of mouse GAPDH cDNA, and Cx43 forward primer 5'-CGTGGAGATGCACCTGAAG-3' and reverse 5'-TGCAGGTGTAGACCGCACT-3' for the amplification of a 129-bp fragment of mouse Cx43 cDNA. PCR was performed using the touchdown temperature cycling system (iCycler, Bio-Rad, Hercules, CA, USA). After an initial denaturing step at $94^{\circ} \mathrm{C}$ for $5 \mathrm{~min}$, amplification for GAPDH and $\mathrm{Cx} 43$ mRNA was performed with 35 cycles at $94^{\circ} \mathrm{C}$ for $30 \mathrm{sec}$ (denaturing), $55^{\circ} \mathrm{C}$ for $1 \mathrm{~min}$ (annealing) and $72^{\circ} \mathrm{C}$ for 1 min (extraction). Electrophoresis of the PCR products was performed in a $1.5 \%$ agarose gel. The gel was stained with GelStar Nucleic Acid Gel Stain (Takara Bio Inc., Shiga, Japan). Densitometry was carried out using an inverted scan image analysis (GDS7500, Funakoshi Co., Tokyo, Japan).

Tumor tissues were minced and homogenized with a homogenizer in ice-cold lysis buffer. Supernatants were collected and used to examine the expression of different proteins by Western blot analysis. Protein concentrations were determined by the Bradford method (Biowave S2100, Funakoshi Co., Tokyo, Japan). Total protein extract $(20 \mu \mathrm{g})$ from the tumors was loaded onto a $10 \%$ SDS-polyacrylamide gel for analysis of the expression level of $\mathrm{Cx} 43$. 3 -actin was used as an internal standard. After electrophoresis, the proteins were transferred to nitrocellulose membranes. Protein expression levels were detected using an enhanced chemiluminescence detection system (Chemdoc, Bio-Rad Laboratories, Tokyo, Japan). The relative density of each protein band on a blot was measured using Quantity One computer software, version 4.5 (Bio-Rad). Molecular sizing was carried out using Rainbow MW markers (Amersham Health Care, Buckinghamshire, UK).

Assessment for hepatic metastases. Microscopic metastastic colonies on the hepatic surface were counted and then fixed with paraformaldehyde. Liver sections stained with hematoxylin and eosin were examined for micrometastasis detection using a microscope.

Statistical analysis. Data were expressed as the means \pm standard deviation. Statistical analyses were performed using the StatView Japanese Edition software package, version 5 (Hulinks Inc., Tokyo, Japan). Different percentiles were calculated from the measured values. The mean values for the pre- and post-treated groups were compared with the mean value of the control group using Tukey's test as a post hoc test after analysis of variance by one-way ANOVA. The level of significance was $\mathrm{p}<0.05$.

\section{Results}

The mean relative weights of the tumors and livers in M5076bearing mice fed with BBIC are illustrated in Fig. 1. Oral BBIC was well tolerated in these experiments without detectable side effects. The relative tumor weights in the 

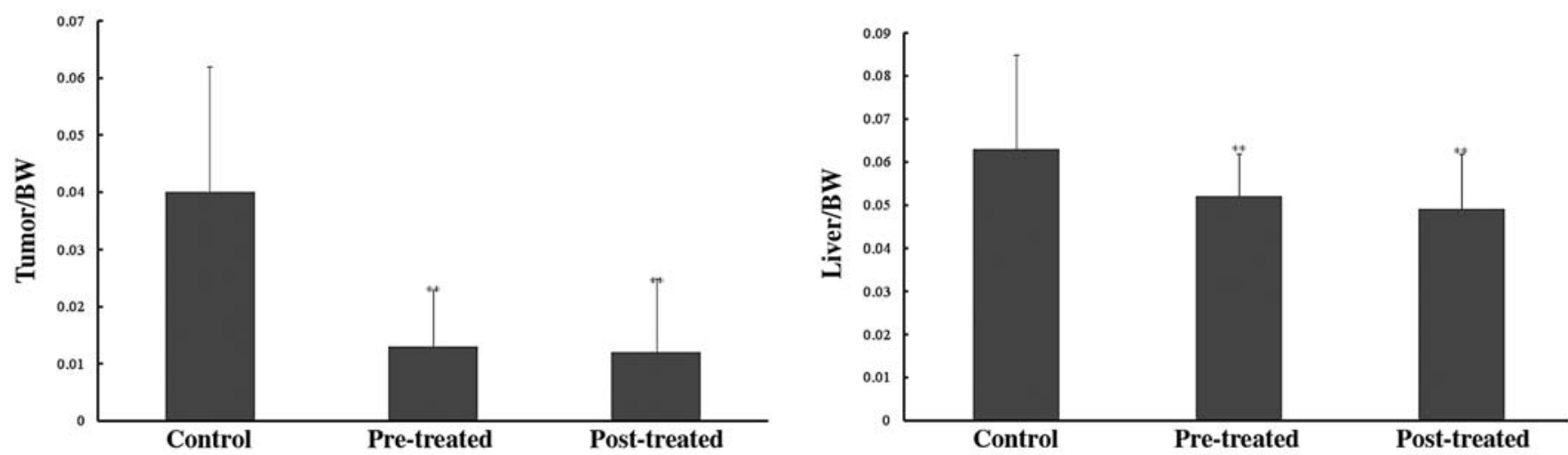

Figure 1. Effect of BBIC treatment on relative tumor (left) and liver (right) weights in M5076-bearing mice. Levels of significance: ${ }^{* *}$ p $<0.01$ versus the control group (Tukey's test). Data are the means \pm SD of 10 mice per group.
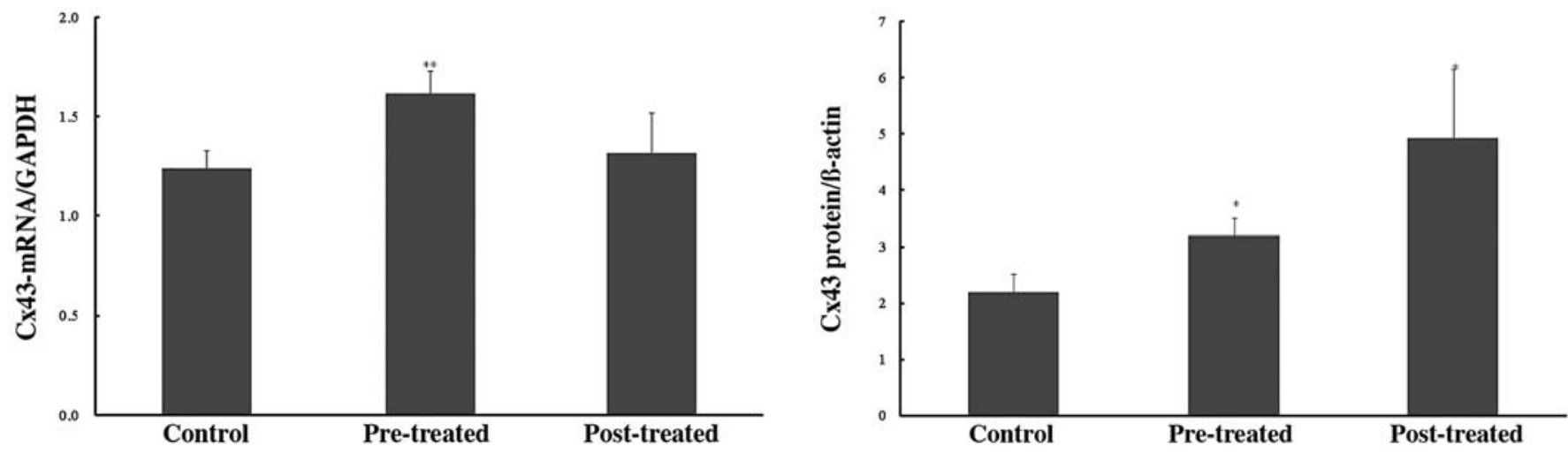

Figure 2. Effect of BBIC treatment on the expression levels of Cx43 mRNA (left) and Cx43 protein (right) in M5076-bearing mice. Levels of significance: ${ }^{*} \mathrm{p}<0.05$ and ${ }^{* *} \mathrm{p}<0.01$ versus the control group (Tukey's test). Data are the means \pm SD of 10 mice per group.

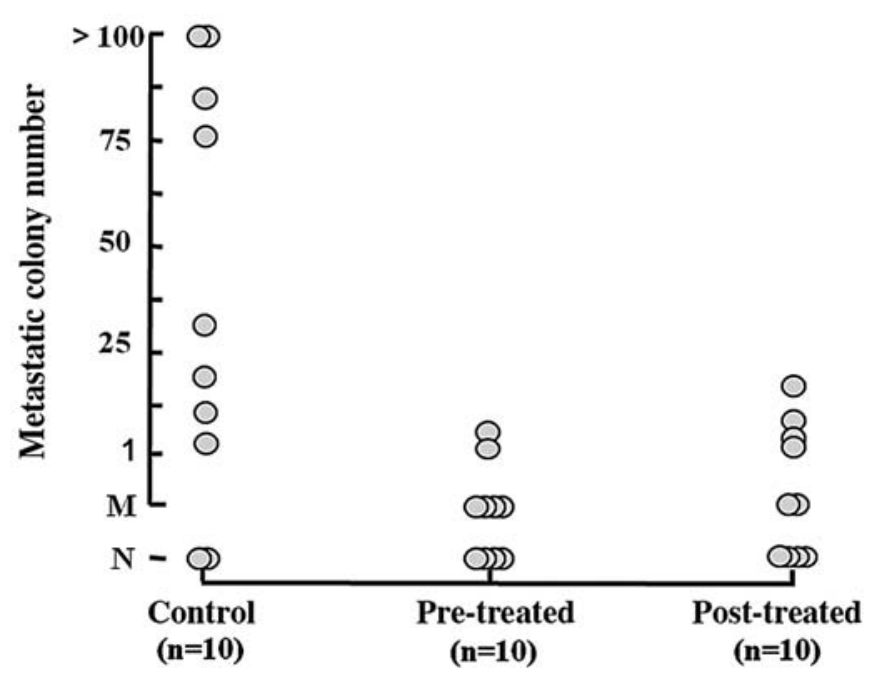

Figure 3. Colonization numbers of the liver surface and sections in M5076bearing mice. $\mathrm{N}$, metastasis not detected; $\mathrm{M}$, microscopically evident tumor foci.

control, pre-treated and post-treated groups were $0.040 \pm 0.022$, $0.013 \pm 0.010$ and $0.012 \pm 0.013$, respectively. In the pre- and post-treated groups, the relative tumor weight was significantly reduced by 32.5 and $30.0 \%$, respectively, as compared to the control group $(\mathrm{p}<0.01)$.
Fig. 2 shows the relative densities of $\mathrm{Cx} 43 \mathrm{mRNA}$ and proteins in the tumors obtained from M5076-bearing mice fed with BBIC. The relative densities of Cx43 mRNA (Cx43/ GAPDH density) in the control, pre-treated and post-treated groups were $1.24 \pm 0.09,1.62 \pm 0.11$ and $1.32 \pm 0.20$, respectively. The relative density of Cx43 mRNA was significantly higher in the pre-treated group than in the control group $(p<0.01)$. The relative densities of $\mathrm{Cx} 43$ protein (Cx43/ß-actin density) in the control, pre-treated and post-treated groups were $2.20 \pm 0.33$, $3.20 \pm 0.31$ and $4.93 \pm 1.23$, respectively. The relative densities of $\mathrm{Cx} 43$ protein were significantly higher in the pre- and post-treated groups than in the control group $(\mathrm{p}<0.05)$.

The relative liver weights in the control, pre-treated and post-treated groups were $0.063 \pm 0.009,0.049 \pm 0.004$ and $0.052 \pm 0.003$, respectively. In the pre-treated and post-treated groups the relative liver weight was significantly reduced to 77.7 and $82.5 \%$, respectively, as compared to the control group $(\mathrm{p}<0.01)$. Macroscopic metastatic colonies on the liver were found in $8 / 10,4 / 10$ and 2/10 mice in the control, pre-treated and post-treated groups, respectively (Fig. 3). The median number of macroscopic metastases was $71.4 \pm 97.3,1.0 \pm 2.3$ and $1.9 \pm 3.6$, respectively. These values were significantly lower in the pre- and post-treated groups than in the control group $(\mathrm{p}<0.05)$. Upon histological examination, no microscopic metastasis was detected in $2 / 10,4 / 10$ and 4/10 mice in the control, pre-treated and post-treated groups, respectively. 


\section{Discussion}

In the present study, it was found that relative tumor weight and the rate of liver metastasis were reduced in mice fed BBIC compared with untreated mice. Furthermore, Cx43 mRNA and $\mathrm{Cx} 43$ protein levels were higher in mice fed BBIC than in mice not undergoing BBIC treatment. These findings elucidate the effect of BBIC in the suppression of tumor development, which depends on the restoration of $\mathrm{Cx} 43$ expression, and demonstrated its utility as a therapeutic agent against tumors with low $\mathrm{Cx} 43$ expression levels.

Epidemiological observations during the last century have shown that cancer incidence and mortality vary significantly across regions of the world $(16,17)$. Populations whose major diets are centered on plant foods tend to have lower rates of cancer (18). BBI derived from soybeans has been studied extensively as an anti-carcinogenic protease inhibitor, as many protease inhibitors suppress carcinogenesis (19). Several studies have demonstrated the efficacy of BBI against tumors in animal models $(9,11,20)$ and in human Phase IIa clinical trials $(12,14)$. BBI is the only protease inhibitor derived from soybeans that possesses a chymotrypsin inhibitory activity (21). The trypsin inhibitory activities of BBI indirectly inhibit the activation of pro-MMP-1, -2 and -9 to their active forms (22). Chen et al (9) demonstrated that the chymotrypsin inhibitory activity of BBI specifically inhibits proteasomal chymotrypsin-like activity. Furthermore, one recent study confirmed that BBI induced the up-regulation of $\mathrm{Cx} 43$ expression and suppression of tumor cell growth in vivo and in vitro $(11,23)$. Thus, it is thought that BBI is useful as an anti-tumor drug for inducing the inhibition of tumor cell growth, invasion and metastasis. BBI enters the circulation via the intestinal epithelial cells or the paracellular mechanism (24). Billings et al (24) demonstrated that, $3 \mathrm{~h}$ following ingestion, up to $40 \%$ of ingested BBI was found in the blood stream, and approximately $17 \%$ in the bladder (24). In addition, BBI appears to be non-toxic to humans and animals (25).

In the present study, no apparent changes caused by the administration of BBIC were found. In summary, BBIC has the same anti-carcinogenic profile as purified BBI, and can be used safely and inexpensively in animal experiments. We therefore evaluated the ability of BBIC to inhibit the growth of M5076 ovarian sarcoma by oral administration. The current findings show that tumor weights were significantly reduced in groups treated with BBIC as compared to the control groups. This study provides evidence in favor of the anti-carcinogenic effects of BBI, although no difference in tumor weight was found between the pre-treated and post-treated groups. These results were interpreted in terms of an inadequate period of pretreatment, indicating that the cancer chemopreventive effect of BBI cannot be detected with a long-term administration period of 3 weeks, as in this experiment.

Most tumor cells have a reduced ability to communicate between themselves and/or with surrounding normal cells, which confirms the importance of functional gap junction intercellular communication (GJIC) in growth control (26). The inhibition of GJIC between adjacent cells has therefore been postulated as an important event taking place during the promotional stage of cancer (27). More recently, direct evidence for the role of GJIC in tumor suppression has been obtained by the transfection of $\mathrm{Cx}$ genes into noncommunicating tumor cells (27-29). Our previous study demonstrated that the anti-carcinogenic effect of BBI-induced negative growth control is caused by increases in p27 accumulation by expression of $\mathrm{Cx} 43$ as a GJIC-dependent pathway (11). The effects of the induction of Cx43 mRNA synthesis and the inhibition of $\mathrm{Cx} 43$ protein degradation contribute to the stabilization of the gap junction (GJ), while reinforcement of the tumor-suppressive effect of $\mathrm{Cx} 43$ is based on the formation of the GJ (30). Thus, the effect of BBI on Cx43 may be effective in establishing cancer chemoprevention and therapy based on $\mathrm{Cx}$ functions. Based on these criteria, BBI appears to be a potential agent for cancer chemoprevention and therapy, as it has been shown to stimulate the biosynthesis of $\mathrm{Cx} 43$ and to suppress the degradation of $\mathrm{Cx} 43$ protein (23). In this study, BBIC was observed to increase the levels of Cx43 mRNA and to stabilize Cx43 protein. Cx43 protein levels were significantly elevated in the pre- and post-treated groups in comparison with the control group, whereas no apparent increase in Cx43 mRNA levels was found between the posttreated and control groups. This is thought to be due to the higher chymotrypsin inhibitory activity of BBIC compared to BBI. Indeed, this activity is thought to be important in inhibiting the degradation of $\mathrm{Cx} 43$ by proteasomes (23). It has been assumed that BBIC preferentially acts as an inhibitor of Cx43 degradation because of its high chymotrypsin inhibitory activity. Recent data makes it increasingly clear that GJIC contributes to the inhibition of metastatic outgrowth in breast and prostate cancer $(31,32)$. Therefore, in the present study the inhibition of hepatic metastasis was investigated in M5076bearing mice. The results indicate that BBIC is useful for the treatment of tumors with suppressed $\mathrm{Cx} 43$ expression, which results in the reduction of metastasis and tumor growth in a Cx43-dependent manner.

The M5076 tumor line, a histiocytic sarcoma that arose spontaneously in the ovary of a C57BL/6 mouse, displays functional and ultrastructural characteristics indicating that it is of macrophage origin (33). Cx43 expression and the formation of the GJ have been found in macrophages (34). Regardless of the site of the primary tumor, this neoplasm rapidly and preferentially metastasizes to the liver. This metastatic behavior is strongly supportive of the organ-microenvironment requirement, as suggested by the 'seed and soil' hypothesis. Mere arrest and retention of tumor cells in a specific organ is not necessarily followed by growth and tumor development (35). Macroscopic examination of the extracted livers indicated that spontaneous metastasis differs significantly between the BBI-treated groups and the control group. BBIC treatment induced a significant reduction in the number of macroscopic metastatic colonies on the liver, and also significantly reduced the relative liver weight, which has been demonstrated to be indicative of hepatic metastasis in M5076 cells (15). Moreover, upon histological examination, metastasis was not detected in 4/10 mice in the pre-treated and post-treated groups together, compared with $2 / 10$ mice in the control group. The metastatic colonies in the control group were large visible foci, but in the treated groups foci were not visible to the naked eye (i.e., microscopic). This observation is construed as a delay in the beginning of metastasis or a suppression of secondary tumor growth in the treated groups. In this study, inhibition of the 
number of metastatic colonies was possibly reflected by the suppression of primary tumor growth. However, it has been suggested that the inhibitory effect had on the primary tumor does not also inhibit hepatic metastasis of the M5076 tumor (15). It is well known that tumor cells secrete serine proteases, which play important roles in tumor cell invasion and metastasis. Therefore, the effect of serine protease inhibitors on tumor metastases was a focal point (26). Since BBI reduced the activities of the various serine proteases, it may function as an anti-metastatic agent in addition to acting as a tumor preventive agent. Although it has been reported that BBI does not suppress the pulmonary metastasis of murine Lewis lung carcinoma 3LL cells or peritoneal disseminated metastasis of human ovarian cancer HRA cells (26), investigation of the anti-metastatic effect of BBI on various types of tumors, such as mesenchymal neoplasms, is necessary.

Overall, we conclude that the chemopreventive, anti-tumor and anti-metastatic effects of BBIC are based on both the induction of $\mathrm{Cx} 43$ mRNA and the stabilization of $\mathrm{Cx} 43$ protein in M5076 ovarian sarcoma. This report has demonstrated that BBIC is useful as a chemopreventive and anti-carcinogenic agent, and is dependent on the restoration of $\mathrm{Cx} 43$ expression.

\section{Acknowledgements}

This study was supported by a research grant from the Fuji Foundation for Protein Research, a research grant on Human Science Focusing on Drug Innovation from the Japan Human Sciences Foundation (KH21012) and a grant-in-aid for Science Research from the Ministry of Education, Culture and Sciences of Japan (no. 16580259) to T. Yano.

\section{References}

1. Du X, Beloussow K and Shen WC: Bowman-Brink protease inhibitor and its palmitic acid conjugate prevent 7,12-dimethylbenz $[\alpha]$ anthracene-induced transformation in cultured mouse mammary gland. Cancer Lett 164: 135-141, 2001.

2. Bowman DE: Differentiation of soybean antitryptic factors. Proc Soc Exp Biol Med 63: 547-550, 1946.

3. Birk Y: Purification and some properties of a highly active inhibitor of trypsin and alpha-chymotrypsin from soybeans. Biochim Biophys Acta 54: 191-197, 1961.

4. Kennedy AR: The Bowman-Birk inhibitor from soybeans as an anticarcinogenic agent. Am J Clin Nutr 68 (Suppl 6): 1406-1412, 1998.

5. Birk Y: The Bowman-Birk protease inhibitor: trypsin- and chymotrypsin-inhibitor from soybeans. Int J Pept Protein Res 25: 113-131, 1985.

6. Losso JN: The biochemical and functional food properties of the bowman-birk inhibitor. Crit Rev Food Sci Nutr 48: 94-118, 2008.

7. Tikhonova TV, Gladysheva IP and Larionova NI: Retardation by the soybean Bowman-Birk inhibitor of elastin hydrolysis catalyzed by leukocyte proteinases. FEBS Lett 362: 225-228, 1995.

8. Kennedy AR: Chemopreventive agents; protease inhibitors. Pharmacol Ther 78: 167-209, 1998.

9. Chen YN, Huang SC, Lin-Shiau SY and Lin JK. Bowman-Birk inhibitor abates proteasome function and suppresses the proliferation of MCF7 breast cancer cells through accumulation of MAP kinase phosphatase-1. Carcinogenesis 26: 1296-1306, 2005.

10. Zhang YW, Nakayama K, Nakayama K and Morita I: A novel route for connexin 43 to inhibit cell proliferation: negative regulation of s-phase kinase-associated protein (skp2). Cancer Res 63: 1623-1630. 2003.

11. Suzuki K, Yano T, Saduzka Y, Sugiyama T, Seki T and Asano R: Restoration of connexin 43 by Bowman-Birk protease inhibitor in M5076-bearing mice. Oncol Rep 13: 1247-1250, 2005.
12. Malkowicz SB, McKenna WG, Vaughn DJ, et al: Effects of Bowman-Birk inhibitor concentrate (BBIC) in patients with benign prostatic hyperplasia. Prostate 48: 16-28, 2001.

13. Armstrong WB, Kennedy AR, Wan XS, Atiba J, McLaren CE and Meyskens FL: Single-dose administration of Bowman-Birk inhibitor concentrate in patients with oral leukoplakia. Cancer Epidemiol Biomarkers Prev 9: 43-47, 2000.

14. Armstrong WB, Kennedy AR, Wan XS, et al: Clinical modulation of oral leukoplakia and protease activity by Bowman-Birk inhibitor concentrate in a phase IIa chemo-prevention trial. Clin Cancer Res 6: 4684-4691, 2000.

15. Sugiyama T and Sadzuka Y: Combination of theanine with doxorubicin inhibit hepatic metastasis of M5076 ovarian sarcoma. Clin Cancer Res 5: 413-416, 1999.

16. Kelloff GJ, Crowell JA, Steele VE, et al: Progress in cancer chemoprevention: development of diet-derived chemopreventive agents. J Nutr 130: 467S-471S, 2000.

17. Stewart BW and Coates AS: Cancer prevention: a global perspective. J Clin Oncol 23: 392-403, 2005.

18. Munoz de Chavez M and Chavez A: Diet that prevents cancer: recommendations from the American Institute for Cancer Research. Int J Cancer 11: S85-S99, 1998.

19. Kennedy AR: Overview: anticarcinogenic activity of protease inhibitors. In: Protease Inhibitors as Cancer Chemopreventive Agents. Troll W and Kennedy AR (eds). Plemium Press, New York, pp9-64, 1993.

20. Kennedy AR, Kritchevsky D and Shen WC: Effects of spermineconjugated Bowman-Birk inhibitor (spermine-BBI) on carcinogenesis and cholesterol biosynthesis in mice. Pharm Res 20: 1908-1910, 2003.

21. Yavelow J, Collins M, Birk Y, Troll W and Kennedy AR: Nanomolar concentrations of Bowman-Birk soybean protease inhibitor suppress X-ray induced transformation in vitro. Proc Natl Acad Sci USA 82: 5395-5399, 1985.

22. Crass RA and Morgan RGH: The effect of long-term feeding of soybean flour diets on pancreatic growth in the rat. Br J Nutr 47: 119-129, 1982.

23. Saito T, Sato H, Virgona N, et al: Negative growth control of osteosarcoma cell by Bowman-Birk protease inhibitor from soybean; involvement of connexin 43. Cancer Lett 253: 249-257, 2007.

24. Billings PC, St. Clair WH, Maki PA and Kennedy AR: Distribution of the Bowman-Birk protease inhibitor in mice following oral administration. Cancer Lett 62: 191-197, 1992.

25. Losso JN, Munene CN and Bansode RR: Inhibition of metalloproteinase- 1 activity by the soybean Bowman-Birk inhibitor Biotechnol Lett 26: 901-905, 2004.

26. Kobayashi H, Fukuda Y, Yoshida R, et al: Suppressing effects of dietary supplementation of soybean trypsin inhibitor on spontaneous, experimental and peritoneal disseminated metastasis in mouse model. Int J Cancer 112: 519-524, 2004.

27. Fujimoto E, Satoh H, Negishi E, et al: Negative growth control of renal cell carcinoma cell by connexin 32: possible involvement of Her-2. Mol Carcinog 40: 135-142, 2004.

28. Berke G, Krutovskikh V and Yamasaki H: Connexin 37 gene is not mutated in lung carcinomas 3LL and CMT. Cancer Lett 195: 67-72, 2003

29. Hirai A, Yano T, Nishikawa K, et al: Down-regulation of connexin 32 gene expression through DNA methylation in a human renal cell carcinoma cell. Am J Nephrol 23: 172-177, 2003.

30. Salameh A and Dhein S: Pharmacology of gap junctions. New pharmacological targets for treatment of arrhythmia, seizure and cancer? Biochim Biophys Acta 1719: 36-58, 2005.

31. Kennedy AR and Wan XS: Effects of the Bowman-Birk inhibitor on growth, invasion, and clonogenic survival of human prostate epithelial cells and prostate cancer cells. Prostate 50: 125-133, 2002.

32. Steeg PS: Metastasis suppressors alter the signal transduction of cancer cells. Nat Rev Cancer 3: 55-63, 2003.

33. Talmadge JE and Hart IR: Morphologic studies on a murine reticulum cell sarcoma (histiocytic sarcoma) of histiocytic origin and its metastases. Vet Pathol 20: 342-352, 1983.

34. Eugenin EA, Branes MC, Berman JW and Saez JC: TNF- $\alpha$ plus INF- $\gamma$ induce connexin43 expression and formation of gap junctions between human monocytes/macrophages that enhance physiological responses. J Immunol 170: 1320-1328, 2003.

35. Hart IR, Talmadge JE and Fidler IJ: Metastatic behavior of a murine reticulum cell sarcoma exhibiting organ-specific growth. Cancer Res 41: 1281-1287, 1981. 